\title{
Method for detecting eye misalignment based on movement near the center of the pupil
}

\author{
Noriyuki Uchida ${ }^{1,2}$ \\ ${ }^{1}$ Kyushu University of Health and Welfare \\ 1714-1 Yoshino-machi, Nobeoka-City, Miyazaki, Japan \\ Kayoko Takatuka², Hisaaki Yamaba², Masayuki Mukunoki², Naonobu Okazaki² \\ ${ }^{2}$ University of Miyazaki \\ 1-1 Gakuen Kibanadai-nishi, Miyazaki-City, Miyazaki, japan \\ E-mail: n-uchida@phoenix.ac.jp
}

\begin{abstract}
Only ophthalmologists and orthoptists are eligible to diagnose misalignment of the eyes amid persistent understaffing. We automated the traditional eye misalignment evaluation (Cover-test) in our earlier studies and developed a technique to distinguish the misaligned eyes from its behavior. This method, however, had a problem with the vertical eye movements being affected by the eyelids and eyelashes, which prevented thorough detection. Therefore, by observing only the movement near the center of the pupil, where it is less likely to be disturbed by the eyelids and eyelashes, we arranged an alternative method to recognize anomalies. Based on this process, we achieved an inspection system and carried out a performance experiment. We confirmed that we could observe not only horizontal motion, but also that of the vertical eye.
\end{abstract}

Keywords: eye position examination, cover test, strabismus/ heterophoria, compact camera

\section{Introduction}

As misalignment of the eyes, such as strabismus and heterophoria, causes defective binocular vision and eyestrain, early detection and training are required. Strabismus in children, in particular, is a risk factor that causes amblyopia. One of the research subjects of pediatric ophthalmology is the diagnosis of strabismus at an early age and the start of treatment. ${ }^{1}$ Only qualified personnel such as ophthalmologists and orthoptists can diagnose eye misalignment, despite the ongoing shortage of skilled personnel. Therefore, it is significant to develop a method to verify the alignment of the eye without trained staff. ${ }^{1,2}$

There are two types of eye position examination: qualitative tests and quantitative tests. Specifically, the qualitative test investigates abnormalities, while the quantitative test measures the magnitude of the ocular deviation. While there are research reports on methods for measuring the amount of ocular deviation, ${ }^{2}$ this approach only works for exotropia, as it is easy to assess the eye location during the measurement. We also explored the idea of using the current eye-tracking system as an alternative tool. With this method, we considered it necessary to plan various parameters for the application site and light to identify the slightest eye movements that suggest strabismus and heterophoria. After the recent development of medical image diagnostic systems, we explored the possibility of creating a machine learning-based detection system based on continuous pictures of eye movements during the examination. We found it arduous to collect study data because the ocular inspection has never been a procedure for image diagnosis before.

As a result, our research developed a basic inspection system capable screening strabismus and heterophoria

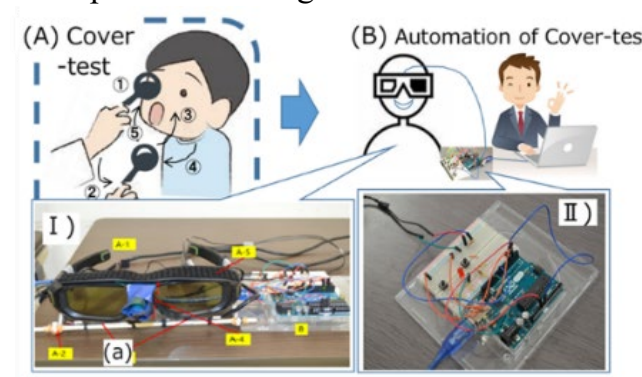

(C) ocular malalignment detection process

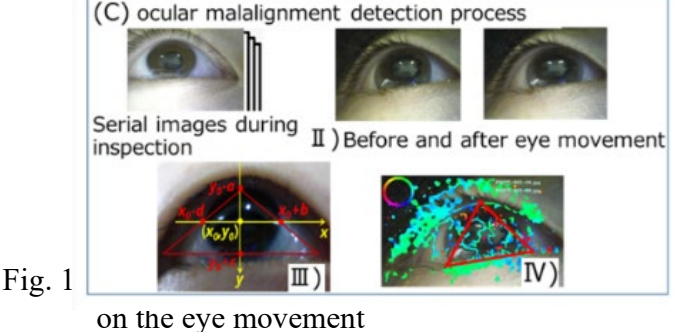

based

(C) The 2021 International Conference on Artificial Life and Robotics (ICAROB2021), January 21 to 24, 2021 
screening without restrictions. We automated the inspection process of the traditional qualitative inspection form, Cover-Test (Fig.1-(A)) (Fig.1-(B)-I, II), ${ }^{1}$ and established and systematized a procedure for the detection of eye movement abnormalities. (Fig.1-(C)-I to IV). Cover-test is an inspection method to decide tropia or heterophoria by the subject focusing on an object. We determine it tropia if the exposed eye moves when the other eye is covered. If the covered eye moves as it is exposed, we judge it heterophoria.

The conventional approach is to cover-uncover the eyes with an occluder and evaluate abnormality by visual inspection (Fig.1-(A)). In the previous analysis, we automated the cover test as follows. With the method we originally developed, we extracted the area for anomaly detection from the moving image around the eye (Fig.1(C)-III). Then, using optical flow technology, ${ }^{3}$ we developed a series of processes that detect eye movement (Fig. 1-C-IV) and assess abnormality., ${ }^{4,5}$ During the test, we took the moving picture around the eye with an ultracompact camera (Fig.1-B-I-(a)) mounted within the left and right lenses. Eye movements displaying signs of eye misalignment occur in approximately 7 to 8 seconds immediately after being covered or exposed. Therefore, we extracted a series of photos taken at a time interval of about 0.2 seconds during that span. However, due to the influence of eyelids and eyelashes, we were unable to observe the vertical movements of the eye.

Vertical strabismus/heterophoria does not have as many cases compared with horizontal strabismus. Early-stage diagnosis is crucial, however, as it can be a sign of severe disorders such as cerebral palsy and heart failure. Also, the number of cases has increased over the past few years due to an aging population. ${ }^{6}$ Therefore, there is considerable significance in developing a system for detecting strabismus and heterophoria in the vertical direction

\section{Research purpose}

The goal of this study was to develop an alternative method for detecting eye misalignment to distinguish not only horizontal but also vertical eye movements. Based on the issues of the previous study, we devised a technique to diagnose disorders by monitoring only the actions near the center of the pupil, where eyelids and eyelashes are less likely to affect the pupil. Section 3 explains the proposed method, Section 4 introduces the performance evaluation experiments of the proposed method, and Section 5 summarizes them.

\section{Proposal method}

We use an ultra-compact infrared camera (referred to as an "infrared camera") to take pictures of the center of the pupil and extract its contour. At the beginning of the test, we refer to the location of the pupil center as the "reference point"(described later in 3.3). Focusing on the dispersion of the distance from the reference point, we find that we can detect strabismus and heterophoria in both directions, both horizontally and vertically, and abnormality.

The following explains the three processes in order.

(1) the extraction of the pupil area (Fig.2-I, II), (2) the measurement of the pupil center coordinates (Fig.2-III), and (3) the determination of the abnormality.

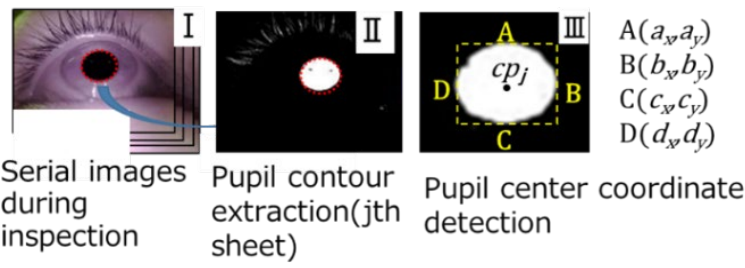

Fig. 2. process of pupil center detection

\subsection{Extraction of the pupil area}

First, we tried to remove the pupil contour with the existing ellipse fitting method. ${ }^{7}$ But we could not extract stable enough to detect the slightest twitch of the eye showing signs of strabismus and oblique location. We, therefore, use the color identification function based on the RGB color model given in OpenCV as another tool. We found that we can extract the pupil contour by setting the RGB values from $(0,0,0)$ to $(15,15,15)$.

\subsection{Calculation of pupil center coordinates}

Fig.2- III shows the coordinates of the extracted pupil area. The y coordinate, where the position coordinate is maximum, is $\mathrm{A}\left(a_{x}, a_{y}\right)$, and the minimum coordinate is $\mathrm{C}\left(c_{x}, c_{y}\right)$. The position coordinate where the $\mathrm{x}$ coordinate is maximum is $\mathrm{B}\left(b_{x}, b_{y}\right)$, and the minimum is $\mathrm{D}\left(d_{x}\right.$, $\left.d_{y}\right)$. We calculate the pupil central coordinate $\mathrm{cp}$ as follows.

$$
c p_{j}=\left(d_{x}+\frac{b_{x}-d_{x}}{2}, \quad a_{y}+\frac{c_{y}+a_{y}}{2}\right)(1)
$$




\subsection{Anomaly Evaluation}

In the previous analysis, we used the real automatic Cover-Test apparatus (Fig.1-(B)) to assess the abnormality of constant eye movements. We found it possible to evaluate anomaly by checking the eye movements seen continuously in the same direction, in the photographs taken at 0.2 -second intervals for around 7 to 8 seconds immediately after the cover/uncover: specifically, the 4 th to 10 th pictures. Using the pupil center location at the start of the test as a reference point, we found that the distance difference between this reference point and the pupil center obtained at each time point during the test estimate the degree of abnormality. Our initial assumption was that the degree of the abnormality would correspond with the absolute distance of eye movement.

However, the findings of the study did not show any correlation. The gap between the eyes and the location of the infrared camera differs between subjects. From the above, we set the measurement parameter based on the variance of the distance from the reference point of the pupil center and carried out an abnormality judgment based on these evaluation criteria.

\section{Setting evaluation criteria}

We achieve a series of transitions of the pupil center coordinates $c p_{j}=\left(\mathrm{x}\left(c p_{j}\right), \mathrm{y}\left(c p_{j}\right)\right)$ from a series of image data (referred to as a test image) taken from around the eye. $(1 \leqq j \leqq n$ and $j$ is the serial number sequence assigned to the test image.) Next, we set the central coordinate $c p_{1}$ taken as the reference point from the first sheet of a series of test images. The distance from the reference point of the center coordinates $c p_{j}(2 \leq j \leq \mathrm{n})$ on the second and subsequent sheets is measured in horizontal and vertical directions. We obtain the average horizontal direction $a_{x}$ and the average vertical direction $a y$, and the variances: $s_{x}^{2}, s_{y}^{2}$.

$$
\begin{aligned}
s^{2} x & =\frac{1}{n} \sum_{n=1}^{n}\left(\left(\left|x\left(c p_{j}\right)-x\left(c p_{1}\right)\right|\right)-a_{x}\right)^{2} \\
a_{x} & =\frac{1}{n} \sum_{j=1}^{n}\left(\left|x\left(c p_{j}\right)-x\left(c p_{1}\right)\right|\right) \\
S^{2}{ }_{y} & =\frac{1}{n} \sum_{n=1}^{n}\left(\left(\left|y\left(c p_{j}\right)-y\left(c p_{1}\right)\right|\right)-a_{y}\right)^{2} \\
a_{y} & =\frac{1}{n} \sum_{j=1}^{n}\left(\left|y\left(c p_{j}\right)-y\left(c p_{1}\right)\right|\right)
\end{aligned}
$$

Through the above method, we have achieved the variances of all the collaborators to produce the study data and the correct judgment results of each individual with the Maddox rod: the precision inspection device. We set the criterion by comparing each variance with the Maddox rod test results. Table 1 indicates the evaluation criteria. Forty-four collaborators contributed to the development of this criterion; the anomalies were 13 in the right position and 36 in the presumed horizontal strabismus or oblique position. The result was: 15 individuals had an angle of deviation in strabismus of $1 \Delta$ (prism), 15 had $2 \Delta$, three had $3 \Delta$, and three $4 \Delta$. To generate pseudo-perspective data on minimal cases of study data for the development of a criterion for vertical strabismus, we followed the specified procedure under the guidance of an expert with the aid of 8 of the 13 collaborators listed above.

Apart from the main contents of the proposed detection method that we mentioned, it was necessary to differentiate between the blinking movement and to exclude the corresponding system from the assessment objective to automate the proposed judgment process completely. And specify the frame when the shutter

\begin{tabular}{|c|c|c|c|}
\hline \multicolumn{2}{|c|}{ vertical direction } & \multicolumn{2}{|c|}{ horizontal direction } \\
\hline $\begin{array}{c}\text { deviation } \\
\text { angle }\end{array}$ & $\begin{array}{l}\text { mean of } \\
\text { variance }\end{array}$ & $\begin{array}{c}\text { deviation } \\
\text { angle }\end{array}$ & $\begin{array}{l}\text { mean of } \\
\text { variance }\end{array}$ \\
\hline$<1 \Delta$ & 1.43 & $<1 \Delta$ & 2.10 \\
\hline $1 \Delta$ & 1.90 & $1 \Delta$ & 2.56 \\
\hline $2 \Delta$ & 4.02 & $2 \Delta$ & 5.63 \\
\hline $3 \Delta$ & 9.66 & $3 \Delta$ & 11.60 \\
\hline $4 \Delta$ & 16.73 & $4 \Delta$ & 21.10 \\
\hline
\end{tabular}
opens and closes. We also automated these processes and modified the automation of the entire detection process.

Table.1. evaluation criteria of abnormal

\section{Verification experiment}

\subsection{Experiment 1}

First, we performed an anomaly judgment using this method for all 20 subjects, compared the judgment results with those of the Maddox rod test, and assessed the system performance by the coincidence rate. We evaluated the outcomes in the following three stages. 
Deviation angles of strabismus larger than $1 \Delta$ and smaller than $3 \Delta$ were abnormal, those larger than $3 \Delta$ were highly abnormal, while those less than $1 \Delta$ were normal.

Table 2-(1) displays the results of the analysis. The notation form is that $\mathrm{V}$ and $\mathrm{H}$ provide the direction of eye movement, and the numerical value after $\mathrm{V}$ and $\mathrm{H}$ represents the angle of strabismus. For example, V0 of V0H6 indicates normality in the vertical direction, and H6 shows that there is a horizontal strabismus/heterophoria of about $6 \Delta$. H0-1 means that the horizontal anomalies are less than $1 \Delta$. Table 2-(1) shows a case, subject B, in which the resulting judgment differs from the correct result of the Maddox rod test resulting in a concordance rate of 95 percent (19/20).

\subsection{Experiment 2}

As mentioned at the beginning, this study aimed to develop a method to detect misalignment in horizontal and vertical eye movements. However, of the 20 subjects, only one case was abnormal in the vertical direction, which did not verify whether we achieved the purpose of this study. Therefore, using pseudo strabismus data, we performed further experimental verification.

With the cooperation with 15 \{A, C, D, E, E, F, G, H, I, $\mathrm{K}, \mathrm{L}, \mathrm{N}, \mathrm{O}, \mathrm{S}, \mathrm{T}\}$ of the 20 subjects whose system judgment result in Experiment 1 was Normal, abnormality degree smaller than $1 \Delta$, we used 20 data (A' to T') randomly selected from the pseudostrabismus data - $8 \times 15$ data: pseudo-strabismus data for 15 subjects, four types $(1 \Delta, 2 \Delta, 3 \Delta, 4 \Delta)$ each in the vertical and horizontal directions - as test data. We performed an abnormality judgment and compared it with the True Value expected when creating pseudo data.

Table 2-(2) shows the judgment results. Four $\left\{D^{\prime}, J^{\prime}, M^{\prime}\right.$, $\left.\mathrm{T}^{\prime}\right\}$ out of 20 cases differed from the expected correct judgment by the system, and the concordance figure was 75 percent $(15 / 20)$.

\subsection{Discussion}

\section{Experiment 1}

As shown in Table 2, the test results of subject B from our system were highly abnormal in both the vertical and horizontal directions. The accurate findings of the Maddox rod test were consistent with the results of the horizontal direction, which were abnormally high, while the results of the vertical direction revealed a significant difference. What we found from the subject B test image was that the overlap between the lower eyelid

and the pupil was substantial. We did not accurately identify the area near the center of the pupil, which could have led to a wrong decision. We will deal with this problem by taking photos of the eyes from the front. As a consequence, the orientation and angle of the camera is the concern.

\section{Experiment 2}

As shown in Table 2, the difference between the outcomes of all 4 cases $\left\{D^{\prime}, J^{\prime}, M^{\prime}, T^{\prime}\right\}$ that did not fit the right device judgment was around $1 \Delta$, which was subtle. We have grouped the causes into three patterns. As in Case B in Experiment 1, we will deal with one out of the four cases by changing the angle of the shooting of the camera.Two cases were irrelevant because they could not construct pseudo strabismus results. The cause of the remaining was a distortion of the pupil contour with the use of hard contact lenses, which we considered irrelevant to this system. Based on the above, we assessed the actual failure to be one out of four.

\section{Table 2. Results}

(1) Experiment 1

\begin{tabular}{|c|c|c|c|c|c|}
\hline subject & $\begin{array}{r}\text { True Value } \\
\text { (Maddox) }\end{array}$ & $\begin{array}{l}\text { System } \\
\text { results }\end{array}$ & subject & $\begin{array}{l}\text { True Value } \\
\text { (Expected } \\
\text { value) }\end{array}$ & $\begin{array}{l}\text { System } \\
\text { results }\end{array}$ \\
\hline A & normal & normal & $A^{\prime}$ & $\mathrm{V} 4 \mathrm{HO}$ & V H.Ab. \\
\hline B & VOH6 & VH H.Ab. & $\mathrm{B}^{\prime}$ & $\mathrm{V} 2 \mathrm{HO}$ & V Ab. \\
\hline $\mathrm{C}$ & normal & normal & $C^{\prime}$ & $\mathrm{VOH} 2$ & $\mathrm{H} \quad \mathrm{Ab}$. \\
\hline$D$ & normal & normal & $D^{\prime}$ & $\mathrm{VOH} 2$ & $\mathrm{VH} A b$. \\
\hline$E$ & normal & normal & $E^{\prime}$ & V3HO & V H.Ab. \\
\hline $\mathrm{F}$ & normal & normal & $\mathrm{F}^{\prime}$ & V3HO & V H.Ab. \\
\hline G & VOHO-1 & normal & $\mathrm{G}^{\prime}$ & $\mathrm{V} 2 \mathrm{HO}$ & V Ab. \\
\hline $\mathrm{H}$ & normal & normal & $\mathrm{H}^{\prime}$ & VOH1 & $\mathrm{H} \quad \mathrm{Ab}$. \\
\hline I & normal & normal & $\mathrm{I}^{\prime}$ & V1H0 & V Ab. \\
\hline 了 & $\mathrm{VOHO}-1$ & normal & $J^{\prime}$ & $\mathrm{VOH} 2$ & $\mathrm{VH} A b$. \\
\hline $\mathrm{K}$ & normal & normal & $\mathrm{K}^{\prime}$ & V4H0 & V H.Ab. \\
\hline $\mathrm{L}$ & normal & normal & $\mathrm{L}^{\prime}$ & V0H1 & $\mathrm{H} \quad \mathrm{Ab}$. \\
\hline$M$ & $\mathrm{VHO}-1$ & normal & $M^{\prime}$ & V1H0 & normal \\
\hline $\mathrm{N}$ & normal & normal & $\mathrm{N}^{\prime}$ & $\mathrm{VOH} 2$ & $\mathrm{H} \quad \mathrm{Ab}$. \\
\hline 0 & normal & normal & $\mathrm{O}^{\prime}$ & V3HO & V H.Ab. \\
\hline $\mathrm{P}$ & VOH5 & H H.Ab. & $\mathrm{P}^{\prime}$ & V1H0 & V Ab. \\
\hline $\mathrm{Q}$ & normal & normal & $Q^{\prime}$ & V0H1 & H Ab. \\
\hline$R$ & V3H12 & VH H.Ab. & $\mathrm{R}^{\prime}$ & V1H0 & V Ab. \\
\hline $\mathrm{S}$ & normal & normal & $S^{\prime}$ & $\mathrm{V} 2 \mathrm{HO}$ & V Ab. \\
\hline $\mathrm{T}$ & normal & normal & $T^{\prime}$ & $\mathrm{V} 2 \mathrm{HO}$ & $\mathrm{VH} A b$. \\
\hline
\end{tabular}

$\mathrm{V}$ : Vertical, $\mathrm{H}$ : Horizontal

Ab.: Abnormal, H.Ab.: Highly Abnormal

(C) The 2021 International Conference on Artificial Life and Robotics (ICAROB2021), January 21 to 24, 2021 


\section{Summary}

To automate the strabismus/heterophoria screening, we have developed an alternative method that can detect not only horizontal but also vertical eye movements.

It is less susceptible to eyelids and eyelashes that make it crucial to recognize the vertical flow of the eye. Specifically, it only senses the motion of the center of the eye where eyelids and eyelashes cannot intervene. With this system, we measured the distance variance from the reference point of each pupil center position and then used it as an assessment criterion to devise a method for detecting anomalies. We have put in place an inspection framework based on the proposed approach and carried out a performance assessment test. First, we set 44 standard inspection data, four pseudo-data forms $\times$ $13=52$, and 96 research data, and evaluation criteria.

We then carried out an anomaly judgment on 40 test data, which is 20 inspection data and 20 pseudo data, and compared the results with the right ones to test the efficiency of the proposed system. As a consequence of the experiment, the success rate of judgment on the data is 85 percent (34/40). However, we found four among the six failures irrelevant to this method because three were false pseudo data, and one was for the use of hard contact lenses. Therefore, the number of actual judgment failures of this system was two out of 40 cases, and the success rate was 95 percent $(38 / 40)$. From the above, we defined the problems with the pupil center detection error for the infrared camera angle of the photograph and explained the future theme. [Acknowledgment] This work was supported by JSPS KAKENHI Grant Number JP19H00505

\section{References}

1. S. Hasebe, Objective Tests of Strabismic Deviation : Basics and Perspective, Journal of the eye 18(9), pp.1105-1110, 2001

2. Y. Takahashi, Y. Shinomiya, T. Noguchi, T. Niida, Study of the occlusion time required for stable fusion-free position by using an eye-tracking system, Folia Japonica de Ophthalmologica Clinica 9(3), pp.234-237, 2016

3. Y. Yamauchi,T. Yamashita, H. Fujiyoshi, Human Detection Based on Statistical Learning from Image, IEICE TRANSACTIONS Vol.J96-D, No.9, pp.20172040,2013

4. N. Uchida, K. Takatuka, K. Hinokuma, K. Hirata, H. Yamaba, N. Okazaki , Automated cover-uncover test system using active LCD shutter glasses, $23 \mathrm{rd}$
International Symposium on Artificial Life and Robotics (AROB), 2018.

5. N. Uchida, K. Takatuka, K. Hinokuma, K. Hirata, H. Yamaba, N. Okazaki, HMD-based Cover Test System for the Diagnosis of Ocular Malalignment, Artificial Life and Robotics (AROB), Vol.24-3, pp332-337, 2019.

6. A. Oohira, Excyclotorsion and Eye Dominancy in Agerelated Small-angle Vertical Strabismus, Journal of Japanese Ophthalmological Society 123(2) pp.161-166, 2019

7. K. Yokota, K. Murata, Y. Sugaya, K. Kanatani, Accuracy Comparison of Ellipse Fitting: From Least Squares to Hyper-Renormalization, IPSJ SIG Technical Report 111(379) pp.123-130, 2012 